\title{
Learning by doing: una aplicación del Aprendizaje Basado en Proyectos (APB) en la asignatura «Metodología de la enseñanza del inglés»
}

\section{Learning by doing: Implementing Project Based Learning (PBL) in the subject "Methodology of English Teaching"}

YOLANDA MORATÓ

ORCID: https://orcid.org/0000-0001-6540-6042

Universidad de Sevilla

Departamento de Filología

Inglesa (Lengua Inglesa)

ymorato@us.es

Fecha de recepción: 2-7-2019

Fecha de aceptación: 11-7-2019

DOI: http://dx.doi.org/10.12795/9788447221912.047

Pp.: 1083-1108 


\section{Resumen}

Este artículo presenta los resultados de un Ciclo de Mejora de Aula (CIMA) aplicado a la asignatura «Metodología de la enseñanza del inglés» del grado en Estudios Ingleses. Se han planificado, desarrollado y expuesto dos proyectos con los que el alumnado ha ahondado en los principales pilares de la asignatura: 1. el estudio de los principales métodos y enfoques de la enseñanza-aprendizaje del inglés como lengua extranjera y 2. el análisis crítico de las destrezas y componentes en dicho proceso de enseñanza-aprendizaje y el diseño de materiales. Para obtener una evaluación más coherente con los objetivos se ha realizado mediante el Aprendizaje Basado en Proyectos (ABP). Dado que la intención era promover una forma de andamiaje o scaffolding para facilitar la adquisición de contenidos, habilidades transferibles y competencias, el presente análisis se apoya sobre los resultados de dos cuestionarios referidos al segundo proyecto de la asignatura.

Palabras clave: Metodología de la enseñanza del inglés, grado en Estudios Ingleses, docencia universitaria, experimentación docente universitaria, Aprendizaje Basado en Proyectos

\section{Abstract}

This article presents the results of a Classroom Improvement Cycle (CIC) applied to the subject "Methodology of English Teaching", which is part of the degree in English Studies. Two projects have been planned, carried out, and presented orally so that students could delve into the main pillars of the subject: 1 . The study of the main methods and approaches to the teaching and learning of English as a foreign language and 2. The critical analysis of the skills and components in those aspects related to the teaching, learning, and design of materials. For this purpose, the evaluation has been planned through Project Based Learning (PBL). Since the intention was to promote a form of scaffolding to facilitate the acquisition of content, transferable skills and competencies, this analysis is based on the results of two questionnaires referred to the second project of the subject.

Keywords: Methodology of English teaching, degree in English Studies, university teaching, university teaching experimentation, Project Based Learning

Jornadas de Formación e Innovación Docente del Profesorado | № 2 (2019) Esta obra se distribuye con la licencia Creative Commons 


\section{Introducción: contexto docente}

Este análisis surge de la puesta en marcha de un cambio conceptual en torno al sistema de evaluación al que tradicionalmente se ha acogido la asignatura de «Metodología de la enseñanza del inglés» (en adelante MEI), que se imparte como optativa de cuarto curso en el grado en Estudios Ingleses con una carga de 6 ECTS. Como sucede en varias asignaturas del grado, el mayor peso de la nota recaía en los exámenes. No es infrecuente que las pruebas escritas e individuales de las materias de los grados universitarios computen entre un 50\% y un $80 \%$ de la calificación total. Sin embargo, para esta asignatura resultaba un contrasentido que el alumnado recibiese una calificación dentro de esa amplia horquilla porcentual por volcar en el papel los principales datos relativos a los procesos de enseñanza-aprendizaje del inglés como lengua extranjera. En otras palabras, recordar una serie de datos acerca del aprendizaje y la enseñanza de una lengua extranjera no garantiza que la aplicación de dicha teoría se produzca dentro de los parámetros de la eficacia, la eficiencia, la objetividad y la atención a la realidad del aula, que es lo que persigue desarrollar esta asignatura.

El objetivo principal de este Ciclo de Mejora de Aula (en adelante CIMA) era lograr un cambio en la adquisición de contenidos mediante la experimentación directa de los distintos componentes del programa, trasladando el foco del profesor al alumno. Aunque mi anterior CIMA se centró en el temario de una asignatura distinta (Lengua Inglesa I, de primer curso), ya pude observar que a mayor grado de implicación de los alumnos en las actividades propuestas, mejor se adquirían los contenidos y competencias clave y mayor reflexión crítica se producía en el aula. La expresión learning by experience, acuñada en 1938 por el filósofo y pedagogo estadounidense John Dewey, resume bien cómo el principio del aprendizaje a través de la acción y la experiencia se opone a los modelos considerados como 
tradicionales: "to learning from texts and teachers, learning through experience; to acquisition of isolated skills and techniques by drill, is opposed acquisition of them as means of attaining ends which make direct vital appeal" (1938; 2007, p. 19).

Si en otras facultades y disciplinas académicas como Arquitectura, Medicina e Ingeniería, por citar tres ejemplos, cuentan con asignaturas de cuarto curso en las que se prescinde de pruebas finales para poner en práctica todo lo aprendido mediante proyectos con los que se manifieste empíricamente lo adquirido, ¿por qué no hacerlo en una asignatura de índole tan práctica como MEl? Si se atiende al hecho de que la docencia es una de las salidas laborales más habituales entre los egresados de esta titulación, resulta innegable que una asignatura que plantee un aprendizaje a través de la experiencia, guiada con unos objetivos claros y unas herramientas que faciliten el proceso, supone un aliciente para nuestro alumnado.

Modificar el enfoque evaluador de la asignatura no es, sin embargo, una tarea fácil desde ningún punto de vista, pues no se puede intervenir en un área concreta del programa y descuidar el resto de los componentes que son inherentes a las competencias y a su proceso de adquisición. Un cambio de características en la evaluación trae consigo un replanteamiento del temario: no de los contenidos en sí (que ya están fijados en la memoria de la titulación), sino de la manera en que dichos contenidos se trabajan en el aula. Para ello, el primer paso de este replanteamiento fue romper igualmente con la estructura lineal propia de los temarios teóricos y de la enseñanza tradicional. Como se verá en las secciones dedicadas al mapa de contenidos y a su modelo metodológico posible, los temas se han agrupado siguiendo unos criterios de función y funcionalidad para conseguir que se interrelacionen en dos proyectos, en lugar de proponer su estudio como compartimentos estancos. 


\section{Diseño previo del CIMA atendiendo a las características del grupo}

MEI es una asignatura que se imparte en dos grupos, en turnos de mañana y tarde, respectivamente, y cuenta con una matriculación de 35 alumnos por turno. Para organizar este nuevo enfoque, coordiné y pedí a los 70 alumnos que se dividieran en 14 grupos (siete en cada turno, con una composición de entre 4 y 6 estudiantes). Desde la primera semana fueron conscientes de que su tarea principal no consistiría en aprender de memoria una serie de conceptos (algo que, por otra parte, consiguieron sin apenas darse cuenta y con el uso cotidiano de un glosario profesional de nuestra disciplina, el Teaching Knowledge Test Cambridge Glossary, 2015). Al contrario, su labor residiría en argumentar de manera técnica qué aspectos pueden mejorarse en las aulas del siglo XXI y qué otros han influido en el curso de la historia de la enseñanza del inglés como lengua extranjera. Tampoco tendrían que memorizar los datos referentes a las destrezas (su tipología y sus actividades) para volcarlos en un examen, sino cuestionarlos en profundidad para consensuar propuestas propias.

Con los resultados que obtuviesen del análisis de manuales, libros de texto, vídeos y artículos académicos tendrían que poner en práctica soluciones innovadoras y eficientes, tratando de resolver los «puntos ciegos» que se le escapan al docente en su labor diaria. En definitiva, a lo que se aspiraba con estos proyectos era a desarrollar un nuevo plan de trabajo mediante el que no iban a estudiar para aprobar y olvidar, sino para adquirir un conocimiento con el que enfrentarse a los distintos retos de su vida como estudiantes y futuros docentes de una lengua extranjera. Gracias a ambos proyectos también se replantearon su manera de aprender y algunos incluso recuperaron sus intereses por la enseñanza para un futuro a corto y medio plazo. 
En cuanto a la evaluación de la asignatura, el apartado de "Sistemas y criterios de evaluación y calificación" del proyecto docente incluyó de manera explícita desde el comienzo del año académico 2018-2019 dos sistemas de evaluación independientes ( $y$, por tanto, no combinables entre sí) entre los que el alumnado pudo elegir libremente antes de comenzar MEl en el segundo cuatrimestre:

a) una evaluación tradicional, en la que el examen parcial tiene un peso del $30 \%$ y el final de un 70\%. La parte final implica un componente oral expositivo para suplir las interacciones y creación de materiales que no se pudieron producir y observar en el aula de manera sistemática y consistente

b) una evaluación multicomponente que combina un examen parcial (30\%) de conceptos clave con dos proyectos grupales en los que hay elementos escritos, orales, grupales e individuales (35\% cada uno). Para poder acogerse al sistema multicomponente, es necesario que los estudiantes participen en el $80 \%$ de las sesiones presenciales de la asignatura.

Para obtener la máxima puntuación en cada uno de los dos proyectos $(35 \%+35 \%)$ que se propusieron como parte del sistema de evaluación continua, era necesario cumplir con los siguientes requisitos:

- entrega en plazo y forma de todas las fichas que guían los pasos de cada uno de los proyectos (hasta $\mathbf{1 0 \%}$ por proyecto)

- lesson plan comentado (hasta $\mathbf{1 0 \%}$ por proyecto)

- desarrollo y revisión rigurosa de los apartados de componente bibliográfico del proyecto (hasta 5\% por proyecto)

- participación en las exposiciones orales de los hitos pautados en cada uno de los proyectos (hasta $\mathbf{1 0 \%}$ por proyecto) 
- depósito de un trabajo impreso colectivo tras concluir cada uno de los proyectos

- menos de 9 faltas individuales a las distintas sesiones de la asignatura (de un máximo de 45 sesiones durante el cuatrimestre, pues la clase cuenta con 3 semanales)

\section{Mapa de contenidos}

El objetivo principal de este CIMA ha consistido en enseñar a aprender de manera directa y explícita. Para ello, el alumnado, asumiendo el papel de docentes, tenía que implicarse en el desarrollo de los contenidos de la asignatura de manera práctica o, en otras palabras, aplicando cada bloque del temario a problemas reales que surgen en el aula de lengua extranjera. Como se ha adelantado, en el programa de la asignatura los contenidos vienen expresados de forma lineal, divididos en seis grandes temas que deben repartirse a lo largo de quince semanas en el segundo cuatrimestre. En la siguiente ilustración se ofrece de manera visual cómo está organizado cada bloque y los aspectos principales que deben tratarse en cada uno de ellos:

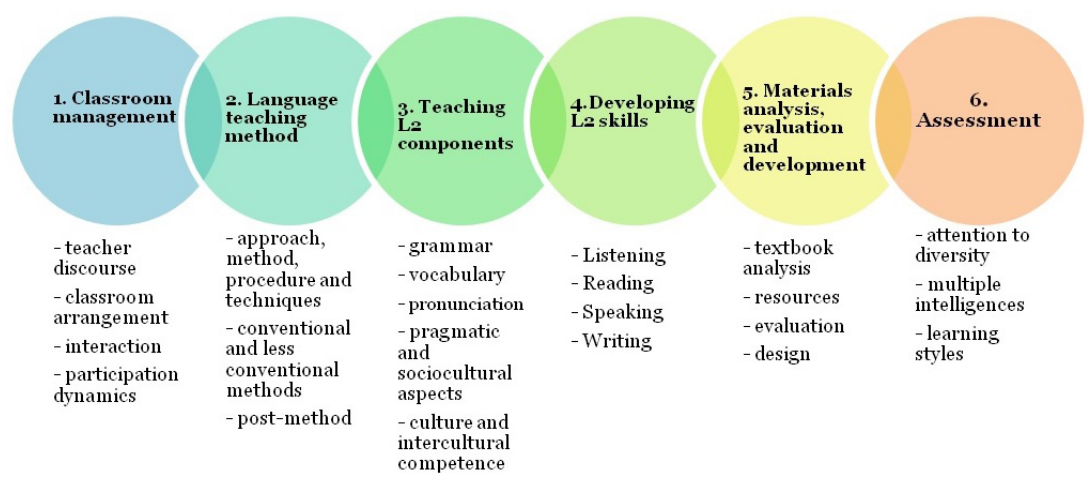

Ilustración 1. Mapa de contenidos según el programa de la asignatura asignatura.jpg

En este punto resultó fundamental realizar un análisis detallado de las competencias específicas que se 
pretendían alcanzar en la asignatura. Con este objetivo, se revisó la guía docente en los periodos estipulados para que reflejara las competencias que se incluyen en la de la memoria de verificación del grado en Estudios Ingleses. Esta revisión fue especialmente beneficiosa para estudiar los posibles proyectos que podían llevarse a cabo en el aula. Entre los factores que hubo que tener en cuenta estaban:

- el tamaño de los grupos (aunque las clases cuentan con una matriculación de 35 estudiantes por grupo, había que planificar proyectos que pudieran trabajarse y exponerse en el aula en horario lectivo, por lo que los grupos debían tener un tamaño medio de entre 4 y 6 participantes)

- el equilibrio teórico-práctico (era necesario encontrar un punto medio entre los contenidos conceptuales y los procedimentales, sin olvidar la inclusión de los actitudinales)

- la concreción de los retos (para llevarlos a cabo en el aula en plazos inferiores a las seis semanas, los proyectos debían tener un objetivo claro que facilitase un amplio abanico de respuestas y, aún así, evitase la aparición de subtemas)

- la formulación de las preguntas de investigación (dado que el alumnado no tiene una formación específica en métodos de investigación, era necesario crear preguntas que fueran acompañadas de instrumentos de investigación y apoyo bibliográfico)

Jornadas de Formación e Innovación Docente del Profesorado | № 2 (2019) Esta obra se distribuye con la licencia Creative Commons Reconocimiento-NoComercial-SinObraDerivada Internacional (CC BY-NC-ND 4.0.) 
Competencias específicas extraídas de la memoria de verificación del grado en Estudios ingleses

\begin{abstract}
E.24. Análisis de las cuestiones básicas relacionadas con el aprendizaje de segundas lenguas, así como de sus implicaciones para la enseñanza de lenguas en el aula.

E.34. Capacidad para diseñar y elaborar materiales formativos y de autoaprendizaje relacionados con los contenidos disciplinares propios del módulo.
\end{abstract}

E.70. Simulaciones sobre los distintos métodos y enfoques de enseñanza de lenguas, por medio de actividades prácticas en clase.

E.71. Desarrollo de la autonomía para seleccionar recursos lingüísticos y metodológicos según los fines a alcanzar.

E.72. Desarrollo del interés por la teoría lingüística y su aplicación a distintos campos, como el estudio de los textos creativos en inglés y la metodología de enseñanza y aprendizaje del inglés.

E.74. Desarrollo del interés por las distintas teorías de enseñanza y aprendizaje de lenguas, en un contexto heterogéneo heredero de tradiciones, métodos y enfoques muy diversos.

E.76. Saber manejar los instrumentos de análisis y búsqueda de información para el estudio de los contextos culturales de los países de habla inglesa.

Para secuenciar y conseguir los objetivos que se encuentran en la memoria de verificación de la titulación (en la tabla 1), los contenidos del programa se dividieron en una estructura biaxial en la que se optó por los dos proyectos que vertebran la asignatura:

1. La gestión del aula y evaluación de la enseñanza aprendizaje de una lengua extranjera (classroom management), atendiendo a los métodos, enfoques, procedimientos y técnicas y el uso de recursos y materiales (temas 1, 2, 5 y 6)

2. La enseñanza-aprendizaje de los distintos elementos y destrezas (components and skills), así como su evaluación, en el aula de lengua extranjera (temas 1, 3, 4 y 6).

Jornadas de Formación e Innovación Docente del Profesorado | № 2 (2019) Esta obra se distribuye con la licencia Creative Commons Reconocimiento-NoComercial-SinObraDerivada Internacional (CC BY-NC-ND 4.0.) 
Aunque a primera vista podría parecer que se han descuidado algunos aspectos del temario en beneficio de otros, una lectura de las relaciones que los seis temas establecen entre sí demuestra que estos dos bloques responden a las preocupaciones metodológicas en la enseñanza-aprendizaje de una lengua extranjera desde numerosos puntos de vista:

1. Los métodos y enfoques que, desde el siglo diecinueve y hasta principios del veinte, se han ido desarrollando en las aulas

2. los materiales que se han empleado para enseñar/ aprender una lengua

3. las destrezas clásicas del inglés (reading, writing, speaking y listening)

4. los componentes (morfología, sintaxis, fonología, semántica y pragmática)

5. la manera en que se evalúan destrezas y componentes

En la siguiente sección se detalla el modelo metodológico y las distintas fases de cada uno de los dos proyectos de la asignatura según los hitos guían cada paso de la investigación desarrollada por los grupos en el aula.

Jornadas de Formación e Innovación Docente del Profesorado | № 2 (2019) Esta obra se distribuye con la licencia Creative Commons 


\section{Modelo metodológico posible y secuencia de actividades programadas}

El modelo que se plantea según los dos proyectos de la asignatura puede ilustrarse así:

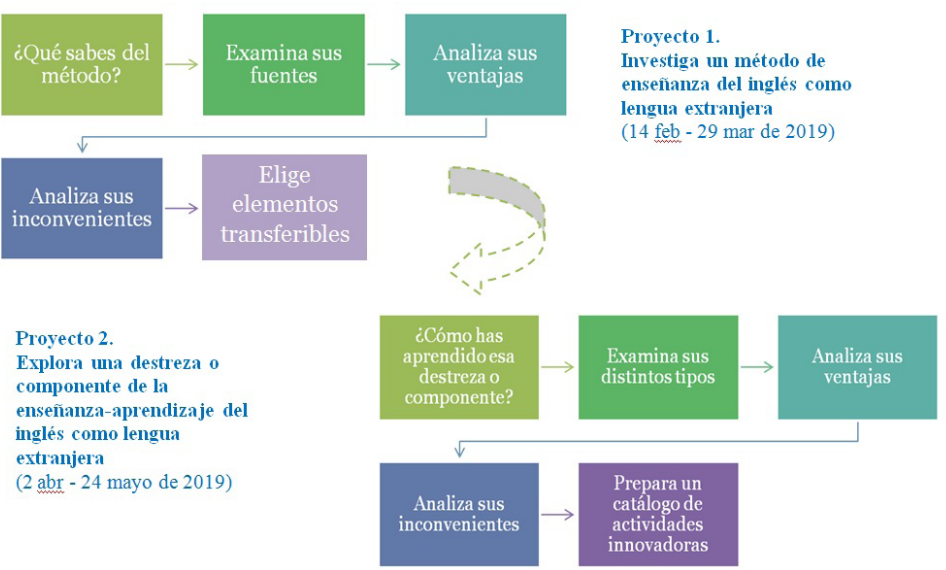

Ilustración 2. Modelo metodológico posible (proyectos y procesos)

Para evitar la sensación de incertidumbre que se produce entre el alumnado en las asignaturas en las que no toman apuntes de manera tradicional y no trabajan con un libro de texto, se les proporcionaron distintos instrumentos de reflexión y guía. Al principio del primer proyecto recibieron un documento de instrucciones sobre los pasos que debían dar y, al final, una lista de comprobación o checklist con la que se autoevaluaron con el objetivo de verificar si había áreas de la primera mitad de la asignatura que no habían asimilado aún. Los veinte puntos clave que recibieron son los siguientes:

\section{I can...}

1. Name, at least, three relevant scholars in the field of Methodology

2. Define different aspects of class management

3. Identify whether a class is teacher-centered or student-centered 
4. Tell the difference between a method and an approach

5. Describe what a procedure is

6. Justify why (or why not) we are living in a post-method era

7. Explain Thornbury's concept of método

8. List, at least, 7 methods from the 20th century

9. Cover the main ideas and tenets of one of these methods

10. Plan a five-part lesson

11. Clarify the functions of each of its parts

12. Create introductory activities as a lead-in to my main activity

13. Make a distinction between rubrics and checklists

14. Specify the main Multiple Intelligences proposed by Howard Gardner

15. Consider Learning styles and their inventories as part of my future teaching assignments

16. Draw the main ideas and key concepts from scholarly articles

17. Provide definitions of, at least, 45 terms from the TKT Cambridge glossary

18. Draft a group project about a method, designing an oral presentation which covers theory and practice

19. Account for different representations of culture in my lesson plan

20. Start thinking about repair strategies and error correction possibilities in my teaching

\section{Selección del marco temporal y conceptual para este CIMA}

Dado que para observar los resultados de este CIMA se decidió escoger únicamente media parte de la asignatura, es decir, un ciclo de 30 horas, el marco temporal y temático elegido fue el correspondiente al segundo proyecto. Por tanto, aunque como ya se ha expuesto, la estructura de la asignatura se modificó por completo desde 
un principio, tanto para el primer como para el segundo proyecto, el análisis pormenorizado de las sesiones se ha realizado a partir de la segunda parte. Se ofrece a continuación la secuencia de actividades, con su descripción y temporización, que se llevó a cabo a lo largo de un periodo de cinco semanas:

\section{Tabla 2}

Tipos de actividades, con su descripción y tiempos, para el proyecto 2

\begin{tabular}{|c|c|}
\hline $\begin{array}{l}\text { Tipo de } \\
\text { actividad }\end{array}$ & Descripción de las actividades \\
\hline $\begin{array}{l}\text { Lluvia de } \\
\text { ideas; revisión } \\
\text { bibliográfica y } \\
\text { extracción de } \\
\text { términos clave }\end{array}$ & $\begin{array}{l}\text { 1. Lectura y debate sobre preguntas de inves- } \\
\text { tigación fundamentales como: ¿Qué es una } \\
\text { destreza? ¿Cuáles predominan en nuestras } \\
\text { clases? ¿Cuáles no están o están en menor } \\
\text { proporción? ¿Por qué? } \\
\text { 2. Extracción preliminar de 5-10 términos clave } \\
\text { a partir de la última edición del TKT Cam- } \\
\text { bridge Glossary (2015). ¿Cómo se definen } \\
\text { términos como task, assessment, rubric o } \\
\text { interaction? } \\
\text { 3. Reflexión sobre cómo se conectan los mé- } \\
\text { todos y enfoques trabajados en el primer } \\
\text { proyecto con los tipos de actividades que } \\
\text { practicamos en el aula de lengua extranjera }\end{array}$ \\
\hline
\end{tabular}

Aplicación práctica de la teoría. Se realiza con una lista de comprobación (checklist) y una ficha (worksheet)

1. ¿Qué grado de representación tiene tu destreza en los manuales de inglés? Redacción de un informe sobre la presencia, desarrollo y características de la destreza sobre la que trabaja el grupo en las distintas partes de un manual o libro de texto

2. Análisis de contenidos clave del componente o destreza a partir de materiales auténticos y experiencia como estudiantes de inglés. ¿Qué define a esa destreza? Clasificar

\section{Tiempos}

Semana 1

Semana 2 en las distintas tipologías

3. Primeros pasos para la transformación de ejercicios tradicionales en tareas y actividades motivadoras para el aprendizaje. ¿Cómo puede motivarse al alumnado con actividades en las que participen y adquieran los contenidos fundamentales de la lengua? 
Debate y redacción de contenidos con fuentes académicas

Desarrollo del pensamiento críticoanalítico
1. ¿Qué aspectos son beneficiosos para la enseñanza de una lengua extranjera? Discusión de las ventajas e inconvenientes de la destreza sobre la que trabaja e investiga el grupo

2. Trabajo con libros de texto y manuales académicos según la destreza asignada

3. ¿Qué bibliografía puede emplearse para consultar actividades innovadoras? ¿Cómo se citan en el trabajo? Familiarización con fuentes de autoridad

1. ¿Qué actividades resultan más beneficiosas para el alumnado? Creación de un catálogo de actividades para el alumnado según la destreza/componente asignado al grupo

2. ¿Con qué términos técnicos justificamos nuestra propuesta? Redacción de términos clave aplicados a las actividades

3. ¿Qué actividades se presentarán en la exposición oral? Selección de las actividades que se incluirán en el trabajo final

Estructuración de la presentación oral

1. ¿En qué orden se presentan las actividades? Planificación de las distintas partes de la exposición

2. ¿Qué recursos adicionales se van a
Semana 3

Semana 4

Semana 5 presentar?

3. ¿Están todas las partes del proyecto? Revisión bibliográfica final

\section{Aplicación del CIMA}

\section{Estructura de las sesiones}

Desde la primera semana de la asignatura, los participantes recibieron dos documentos de orientación cuyo objetivo era guiar la programación de su trabajo a lo largo del cuatrimestre. Se colocaron en la plataforma Blackboard para su consulta:

1. Cuadrante de los distintos grupos e integrantes que participan en la asignatura

2. Esquema del cronograma con los plazos de cada uno de los proyectos y sus fases 
Information about assessment and evaluation

Curso Académico 2018-19

Facultad de Filología - Grado en Estudios Ingleses

Asignatura: Metodología de la Enseñanza del inglés 1830046

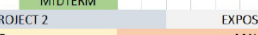

ABR

EXPOS

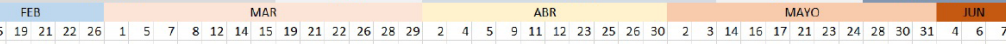

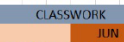

All assessment and evaluation requirements can be checked at: http://www.us.es/estudios/grados/plan 183/asignatura 1830046

Only those students who miss 8 days or fewer will participate in the 2-project plus midterm exam option.

Students selecting the 2-exam option (midterm + final exam) are advised to check their final exams and dates at:

http://filologia.us.es/wo-content/uploads/2016/11/Exam G Ingl 4.pdf

An alternative exam date will be facilitated only in the cases observed at the Normativa Reguladora de la Evaluación y Calificación

de las Asignaturas de la US (BOUS Art. 17).

Ilustración 3. Cronograma de los proyectos de la asignatura (documento 2)

En la primera fase del segundo proyecto los distintos grupos tenían que diseñar y planificar actividades innovadoras (una por cada miembro del equipo) investigando distintos manuales y ofreciendo posibles adaptaciones según la edad, el nivel lingüístico, las características del alumnado y el aula en el que se fueran a impartir. Para esta tarea contaron con todas las clases del mes de abril, en las que se dedicó media sesión (40 minutos) a trabajar con material audiovisual e impreso sobre cada una de las destrezas y la otra media (40 minutos) a debatir en grupo y redactar las soluciones más apropiadas. Ya en mayo, desde el 14 hasta el 24, los siete equipos de cada turno tenían que presentar sus resultados al resto y completar por cada exposición oral que hicieran los miembros de los seis grupos restantes un informe de análisis crítico individual en el que respondieran por escrito a los siguientes aspectos:

1. lo que se plantean incorporar en su futura docencia y por qué

2. factores positivos de cada una de las presentaciones

3. aquello que cada equipo podría mejorar

Fue un acierto haber planificado dos semanas de actividades independientes de los proyectos (colocadas a finales de mayo y principios de junio). Aunque en principio 
las diseñé para incorporar tareas complementarias a los proyectos, las visitas no planificadas de dos docentes del programa Erasmus+ (una en abril y otra en mayo) pudieron adaptarse al cronograma de la asignatura haciendo uso de tres de esos seis días. La Dra. Liliana Piasecka, del Instytut Filologii Angielskiej, de la Uniwersytetu Opolskiego (Opole, Polonia) y la Dra. Adrienn Fekete, de la Pécsi Tudományegyetem (Pécs, Hungría), impartieron tres seminarios sobre cómo introducir la cultura, la creatividad y la poesía en el aula de lengua extranjera. La experiencia fue muy enriquecedora para los distintos elementos del segundo proyecto. Aunque no se incluyen en este artículo, los estudiantes completaron fichas de evaluación de las actividades Erasmus+ en las que plasmaron su alto grado de satisfacción y los aspectos más relevantes para el desarrollo de sus propios proyectos.

\section{Cuestionario inicial-final para hacer un seguimiento de la evolución de los estudiantes en relación con los problemas claves}

Dado que el cambio de sistema de evaluación y, por tanto, la estructuración de los contenidos, suponía una nueva experiencia, también se utilizaron cuestionarios en la fase inicial y final de ambos proyectos con el objetivo de examinar el progreso y medir el aprovechamiento una vez concluidos. Estos cuestionarios eran, a su vez, actividades de evaluación formativa con las que se perseguía medir sus ideas previas (pre-test) y sus ideas adquiridas en el transcurso del proyecto (post-test). Con los resultados del segundo, se han elaborado las escaleras de aprendizaje que se exponen en la sección dedicada a la evaluación del aprendizaje de los estudiantes.

El cuestionario, tanto el inicial como el final, consistía en un reto para el alumnado: durante diez minutos cronometrados cada uno de los grupos debía recoger por escrito 
el máximo número de actividades propias de la enseñanza-aprendizaje de una lengua extranjera que sus miembros fueran capaces de recordar a partir de su experiencia como estudiantes y del conocimiento que hubieran desarrollado en otras asignaturas del grado.

\section{Relato resumido de las sesiones}

Con la llegada del segundo proyecto se repitió la sensación de inseguridad que habían experimentado al comienzo de la asignatura, pues, aunque este segundo reto tendía puentes a las competencias del anterior, se trataba de un proyecto nuevo que se separaba hacia un sendero más creativo: el del diseño de materiales y su justificación técnica dentro del marco docente. Fue en este punto en el que descubrieron, a medida que se iban aportando lecturas, actividades, vídeos, debates, problemas, que nunca habían estudiado una lengua como ellos mismos estaban planteando ahora en el aula. La preocupación fue en aumento a medida que tomaban conciencia del peso que tenían sus aportaciones en la nota, que representaba el 70\% de la calificación final entre los dos proyectos. La mayor parte de las dudas ya se habían disipado al exponer el primer proyecto, pues pudieron ver con sus propios ojos los resultados de su trabajo en grupo. E incluso cuando hicieron el examen de conceptos básicos llegaron a dudar de que pudiera ser tan fácil, pues la realidad es que se ponían a prueba con medio centenar de términos extraídos del Teaching Knowledge Test de Cambridge (2015), el glosario que se utiliza para preparar certificados profesionales para docentes. En el segundo proyecto la incertidumbre dio paso al interés por las notas finales. La conciencia de lo eficaz de este enfoque les había hecho dar un giro: muchos querían rendir ahora todo lo posible para alcanzar la máxima calificación en el modelo formativo de evaluación. 
Aparecían, de nuevo, los viejos modelos: quizás de manera inconsciente querían transformar una evaluación formativa en una sumativa.

El clima de trabajo fue muy satisfactorio, pues los miembros de los distintos grupos ya se habían familiarizado con el proceso y la manera de trabajar en sus respectivos equipos. En cuanto a la actuación docente, la tarea resultó incluso más fácil, porque las dificultades que encontraban los distintos grupos se resolvían con plena confianza durante el transcurso de la clase y las soluciones se compartían con el resto del alumnado. Periódicamente los distintos equipos completaban unas fichas de reflexión sobre los hitos del proyecto, por lo que podían ir meditando sobre las sucesivas etapas del proceso y el avance del aprendizaje.

Entre los aspectos que les crearon un mayor número de dudas siempre estuvo la exposición oral, un hecho que pone de manifiesto que el sistema educativo actual no fomenta entre el alumnado este tipo de dinámicas y no los dota de las herramientas necesarias para que todos, sin excepción, se sientan cómodos presentando los resultados de sus investigaciones frente al resto de la clase. Por último, cabe subrayar que solo una persona se acogió a la evaluación tradicional. Lo hizo porque, debido a sus compromisos laborales, no pudo asistir finalmente al número obligatorio de sesiones de la asignatura, aunque participó en ambos proyectos y aprobó con buena nota la prueba final. 


\section{Evaluación del aprendizaje de los estudiantes}

En este apartado se presentan los resultados obtenidos por los estudiantes en dos cuestionarios separados por cinco semanas. La siguiente ilustración compara sus conocimientos iniciales y finales por medio de dos escaleras de aprendizaje que muestran el número de actividades que fueron capaces de recordar en un breve periodo de tiempo (antes y después de trabajar en sus proyectos).

Para realizar la evaluación del aprendizaje se ha utilizado un mismo reto para todos los grupos en los turnos de mañana y tarde: ¿cuántos tipos de actividades distintas sois capaces de recordar? Se les pide el nombre o la descripción de actividades que se llevan a cabo en el aula para estudiar inglés. Los resultados obtenidos se han clasificado según los niveles comunicativos de dichas actividades. Para una mejor representación gráfica, se han organizado visualmente mediante el formato de Escaleras de Aprendizaje propuestas por Rivero y Porlán (2017, págs. 77-83). Además, para la formulación del reto se han seguido las directrices propuestas por los autores, entre las que destacan las preguntas abiertas e indirectas, que funcionen como anticipadoras de situaciones e intérpretes de resultados "a través de situaciones cotidianas o profesionales de interés" (p. 78).

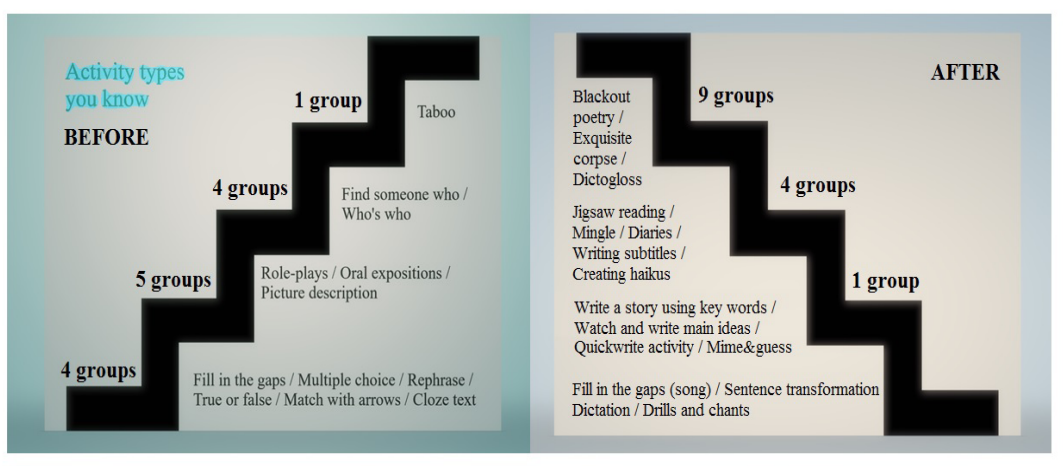

Ilustración 4. Escaleras de aprendizaje a partir del pre-test/post-test

Jornadas de Formación e Innovación Docente del Profesorado I № 2 (2019)

Esta obra se distribuye con la licencia Creative Commons Reconocimiento-NoComercial-SinObraDerivada 
Como puede observarse, en el nivel más alto de la escalera inicial (a la izquierda, Before), solo uno de los catorce grupos nombra una actividad de comunicación libre (la que se asimila al juego de mesa Taboo), mientras que la mayoría se centra en ejercicios de corte tradicional, propios del método Gramática-Traducción, que aún impera en algunas aulas de nuestro país. En la base de la escalera se sitúan, por tanto, todas las actividades características de este método, en las que bien hay que rellenar huecos, bien elegir respuesta entre un sistema de opción múltiple o de Verdadero/Falso. Son todas ellas actividades de respuesta cerrada y encaminadas a preparar exámenes centrados en la demostración de la competencia gramatical en contextos de comunicación muy limitados. No obstante, a medida que se asciende de nivel, comienzan a aparecer actividades más dinámicas, en las que la participación del alumnado cobra un mayor protagonismo, en forma de presentaciones orales o con actividades más lúdicas como el "Quién es quién" y "Encuentra a alguien que...". Sin embargo, el número de grupos que incluye al menos dos actividades de este rango se reduce considerablemente (entre 4 y 5).

En la segunda escalera (a la derecha, After) se refleja justamente lo contrario. La exposición a distintas dinámicas de grupo, a los materiales innovadores y al cotejo de fuentes bibliográficas de los principales especialistas de la materia revela un fuerte impacto entre el alumnado. Aunque entre las propuestas de base siguen apareciendo actividades tradicionales (no en vano son las que han marcado sus vidas como estudiantes de inglés como lengua extranjera), el número de tareas innovadoras es ahora mucho mayor, como también lo es el número de grupos que las incluyen en el post-test. Igualmente, merece destacarse el hecho de que los alumnos utilicen los nombres técnicos de las actividades que proponen, en lugar de descripciones más o menos concretas de los pasos de cada una de ellas, como hacían durante las primeras semanas 
de la asignatura. El nivel de conocimiento que demuestran tras unas semanas es, por tanto, avanzado, tanto en la teoría como en la práctica.

\section{Evaluación del CIMA}

En futuras ediciones de esta asignatura me gustaría conservar los cambios que he introducido en el sistema de evaluación y de agrupación de contenidos mediante proyectos. Ahora que he podido comprobar que es un procedimiento que realmente funciona con los grupos que tenemos en el grado y que no solo supone una experiencia productiva para el alumnado, sino que, además, arroja buenos resultados (tanto en calificaciones como en el nivel de satisfacción de sus participantes), me gustaría incorporar un mayor número de instrumentos para guiar a los equipos en el desarrollo de sus proyectos, reduciendo así mis intervenciones explícitas y favoreciendo un mayor grado de autonomía e implicación entre el alumnado. Mantendría, por tanto, no solo el sistema de evaluación, sino el mapa de contenidos y los dos proyectos, enriqueciendo el enfoque metodológico y su secuencia de actividades.

Entre las principales dificultades que he detectado se encuentra la falta de familiaridad del alumnado con esta metodología. Como resulta obvio, durante las primeras sesiones se sentían muy inseguros ante esta nueva forma de trabajar en clase. A algunos les costó bastante participar a diario, acostumbrados como están a llegar al aula, permanecer en silencio y tomar apuntes. Progresivamente los resultados les demostraron que podían aprender de otra forma. La organización de los distintos apartados haciendo uso de un sistema de preguntas es una opción que repetiría sin duda. Tras leer en la sección "Aprender de los mejores" (Bain, 2007) que las preguntas "desempeñan un papel esencial en el proceso de aprendizaje y en la modificación de los modelos mentales" (pág. 42), me propuse 
incluir al menos tres preguntas semanales que funcionaran como desencadenante para la reflexión y el debate. La visita de dos profesoras del programa Erasmus+, que trabajan siguiendo también este modelo en sus respectivos países, fue muy beneficiosa para confirmar a los distintos grupos que había otras maneras de enseñar y aprender. Los seminarios impartidos por la Dra. Liliana Piasecka (Opole, Polonia) y la Dra. Adrienn Fekete (Pécs, Hungría) les permitieron contar con otras prácticas docentes que poder adaptar en sus proyectos.

En cuanto a los aspectos que he desarrollado y que podrían incorporarse a la práctica docente de cualquier asignatura resalto el cronograma semanal detallado y el sistema organizativo de atención al alumnado. Por un lado, la organización de los contenidos pautados en proyectos, junto con los días dispuestos al final del cuatrimestre para revisar los principales puntos de la asignatura, hicieron posible que pudiéramos incorporar tres sesiones de las dos profesoras Erasmus+, que colaboraron con presentaciones y seminarios creados ex profeso para los proyectos de la asignatura. Me gustaría destacar igualmente el sistema de tutorías que establecí mediante el uso de un programa de software, pues me ha facilitado en gran medida el proceso de orientación y tutorización y me ha permitido ahorrar mucho tiempo en la organización de la atención personalizada.

Gracias al uso de la herramienta Doodle, no he tenido que contestar numerosos mensajes sobre la reserva y disponibilidad de tutorías, sino que los propios alumnos han elegido, mediante cita previa digital, las franjas de $15 \mathrm{mi}-$ nutos que estaban disponibles semanalmente a lo largo de cada mes, siempre por iniciativa propia y según sus horarios y disponibilidad. Aunque Doodle se utiliza precisamente para lo contrario (que sus usuarios marquen todas las franjas que tienen libres para acordar fechas en las que se den coincidencias horarias entre sus 
participantes), este uso alternativo que creé para agilizar las consultas ha sido muy bien recibido por el alumnado, que ha hecho un amplio uso de ellas. 44 personas acudieron solas o acompañadas durante el mes de mayo, facilitando así un seguimiento personalizado del progreso de mis estudiantes y sus proyectos.

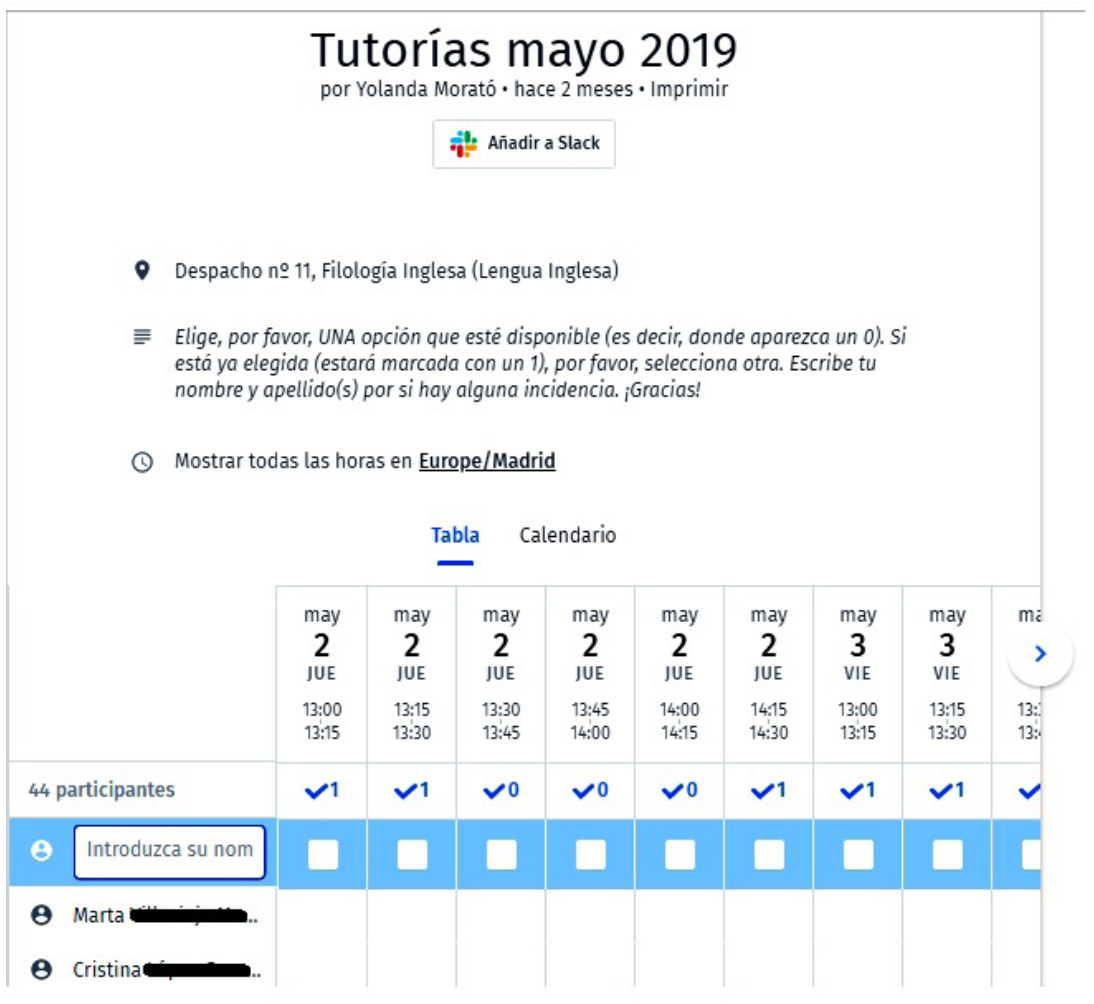

Ilustración 5. Ejemplo de horas de atención a la asignatura con cita previa

Los resultados de este CIMA demuestran que el Aprendizaje Basado en Proyectos ofrece una gran oportunidad para el enseñanza dentro y fuera del aula. Aunque exige un tiempo considerable de planificación y desarrollo de materiales, de búsqueda de planteamientos críticos y dinámicas por parte del docente, el impacto en el alumnado es verdaderamente incuestionable. Se refleja en las altas 
calificaciones que obtiene la mayoría pero, sobre todo, en la participación diaria, en su motivación, en las ganas de aprender y en las inquietudes por avanzar en la materia. A la vista de las conclusiones de este ciclo y una vez recorrido el camino guiado por los principios didácticos que se han expuesto en estas páginas, sería ineficaz volver al sistema anterior.

\section{Bibliografía}

Cambridge English Language Assessment (2015). Teaching Knowledge Test Cambridge Glossary. Recuperado de https://www.cambridgeenglish.org/ Images/22184-tkt-glossary-document.pdf

Dewey, J. (1938/2007). Education and Experience. Nueva York: Simon and Schuster.

Bain, K. (2007). Lo que hacen los mejores profesores universitarios. Valencia: Universidad de Valencia.

Porlán, R. Coord. (2017). Enseñanza universitaria. Cómo mejorarla. Madrid: Ediciones Morata.

Jornadas de Formación e Innovación Docente del Profesorado | № 2 (2019) Esta obra se distribuye con la licencia Creative Commons 
